\title{
Digital image analysis in pathology: Benefits and obligation
}

\author{
Arvydas Laurinavicius $^{\mathrm{a}, \mathrm{b}, *}$, Aida Laurinaviciene ${ }^{\mathrm{a}, \mathrm{c}}$, Darius Dasevicius ${ }^{\mathrm{a}, \mathrm{b}}$, Nicolas Elie $^{\mathrm{d}}$, \\ Benoît Plancoulaine $^{\mathrm{d}}$, Catherine Bor ${ }^{\mathrm{d}, \mathrm{e}}$ and Paulette Herlin ${ }^{\mathrm{d}}$ \\ ${ }^{a}$ National Center of Pathology, Affiliate of Vilnius University Hospital Santariskiu Klinikos, Vilnius, Lithuania \\ ${ }^{\mathrm{b}}$ Faculty of Medicine, Vilnius University, Vilnius, Lithuania \\ ${ }^{\mathrm{c}}$ Institute of Oncology, Vilnius, University, Vilnius, Lithuania \\ ${ }^{\mathrm{d}}$ GRECAN, University of Caen and F. Baclesse Comprehensive Cancer Center, Caen, France \\ ${ }^{\mathrm{e}}$ Pathology Department, F. Baclesse Comprehensive Cancer Center, Caen, France
}

\begin{abstract}
Pathology has recently entered the era of personalized medicine. This brings new expectations for the accuracy and precision of tissue-based diagnosis, in particular, when quantification of histologic features and biomarker expression is required. While for many years traditional pathologic diagnosis has been regarded as ground truth, this concept is no longer sufficient in contemporary tissue-based biomarker research and clinical use. Another major change in pathology is brought by the advancement of virtual microscopy technology enabling digitization of microscopy slides and presenting new opportunities for digital image analysis. Computerized vision provides an immediate benefit of increased capacity (automation) and precision (reproducibility), but not necessarily the accuracy of the analysis. To achieve the benefit of accuracy, pathologists will have to assume an obligation of validation and quality assurance of the image analysis algorithms. Reference values are needed to measure and control the accuracy. Although pathologists' consensus values are commonly used to validate these tools, we argue that the ground truth can be best achieved by stereology methods, estimating the same variable as an algorithm is intended to do. Proper adoption of the new technology will require a new quantitative mentality in pathology. In order to see a complete and sharp picture of a disease, pathologists will need to learn to use both their analogue and digital eyes.
\end{abstract}

Keywords: Quantitative pathology, image processing, quality control, stereology

In the first decade of the twenty first century, pathology entered the era of personalized medicine. Yet, it is difficult to appraise the magnitude of the change that has been brought about by this commonly used concept. In practical terms, it means that pathology procedures will be required to retrieve much more clinically useful information from tissue samples which frequently will be smaller in size. The evolution of pathology is speeding up; the traditional domain of the pathologist providing qualitative diagnostic

*Corresponding author: Arvydas Laurinavicius, National Center of Pathology, Affiliate of Vilnius University Hospital Santariskiu Klinikos, P.Baublio 5, LT-08406 Vilnius, Lithuania. Tel.: +370 5 2720664 (W) and +370 52720044 (F); E-mail: lauar@vpc.lt. information based on "holistic" view of pathology and limited clinical information is rapidly expanding by the increasing demand for semi-quantitative and quantitative evaluation of pathologic features and biomarker expression (Fig. 1). In this context, anatomic pathology is becoming increasingly quantitative or analytical. This poses new requirements for the accuracy and precision of the diagnostic process.

The last decade was also marked by the advancement of virtual microscopy technology enabling high-resolution digitization of the whole microscopic specimen used for diagnosis and research in pathology $[1,2]$. As in many areas of human activity, switching from analogue to digital presents new opportunities to process the signal, thus increasing capacity and 


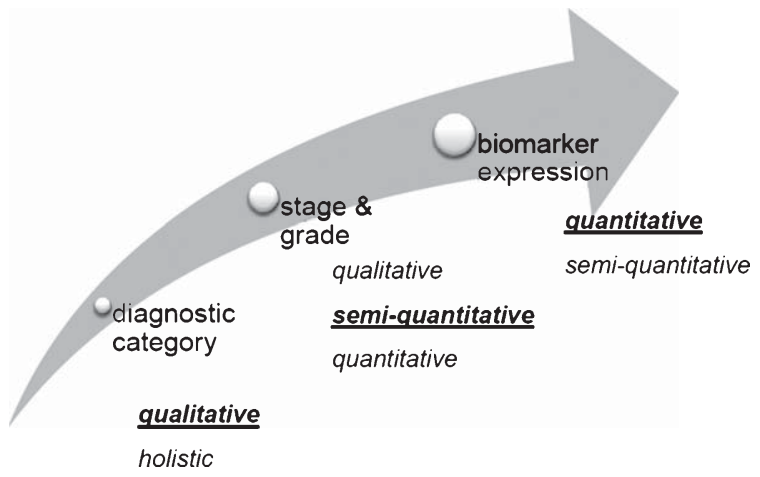

Fig. 1. Evolutionary change in pathology: increasing demand for quantification.

precision of the analysis and retrieving new information invisible to the human eye. In order to solve various tasks of tissue diagnosis and research there is a burst of image analysis applications that follow large field digital microscopy scanning developments [3]. The spectrum of the tasks is broad: from simple morphometry of cells and tissue structures to automated pattern recognition, sub-cellular molecular studies, and multispectral image analysis.

The potential benefits of digital image analysis were explored and largely illustrated during the last three decades. Some innovative applications may revolutionize some areas of pathology [4]. The most extensive adoption of this technology is likely to be in the fields in which improvements in quantification are sorely needed. Specifically, personalized medicine will put the most pressure on pathologists to improve the accuracy and precision of histological grading systems and measurement of biomarker (mainly, immunohistochemical) expression. Clinical studies have shown that it is difficult to control an inter-observer and interinstitutional variability of the assessment of prognostic and predictive markers. This is even more so in pathology practice $[5,6]$. Lack of accurate and reproducible measurements in tissue-based diagnosis is a major obstacle for development of personalized therapies. On the other hand, this demand for accuracy pushes the pathologists to the limits of human capability to standardize their visual perception.

Pathologists use their "trained eyes" to provide useful information for clinical studies and practice. Traditional pathologic diagnosis has been regarded a ground truth. This concept is no longer sufficient in contemporary tissue-based biomarker research and clinical use. Centralised evaluation of biomarkers may improve reproducibility in clinical studies, but it may not provide an accurate measurement of the "real world", due to limitations of human visual perception. Furthermore, guidelines and cut off values derived from these studies are not necessarily reproducible in clinical practice when pathologists worldwide, with variable experiences and settings, are involved [5]. This leads to increased "information noise" and a failure to follow the guidelines appropriately, resulting in accumulation of erroneous evidence. In a strict sense, expectation that pathologists can reliably quantify microscopic features based on visual impressions is outdated. Pathologists could reliably quantify some features under appropriately standardised methodologies but in clinical practice this would result in unrealistic workloads.

Computer vision which is based on the analysis of digital data obtained under controlled conditions, therefore, provides a great opportunity to improve quantification in pathology. The immediate benefits are increased capacity (automation) and reproducibility of the measurements (precision). When the parameters of image analysis are set, the same result will be produced on the same image. However, precision is not a substitute for the measurement of accuracy, which is defined as closeness of agreement between a measured quantity value and a true quantity value of a measurand [7]. In other words, image analysis algorithms may provide precise - reproducible analyses, but they may have their own bias and, therefore, produce inaccurate results. The results of an inaccurate analysis may still be clinically useful in predicting disease or therapy outcomes when validated in clinical studies, however, usefulness should not be confused with accuracy or objectivity [8].

To estimate the accuracy of a measurement, one needs to define and obtain reference values, equivalent to ground truth. The most common approach is to test the agreement of an image analysis algorithm with the pathologists' consensus evaluation, based on interand intra-observer variability in study design [9]. This is a seemingly valid approach since the algorithm is validated against the clinically accepted way of measurement. Nevertheless, the question remains why a more precise and potentially more accurate tool is calibrated against a more variable and semi-quantitative human evaluation. One further step could involve the testing of inter-algorithm variability, especially, if the ground truth may be obtained from an independent 
source like FISH test for HER2 gene amplification (in the case of immunohistochemical measurement of HER2 expression) [10, 11]. However, even in this example HER2 FISH provides only an indirect reference value since biological and technical sources of variation between immunohistochemistry and FISH tests do remain $[12,13]$.

Ideally, to validate and calibrate the image analysis algorithms one should seek the most direct reference value. It should answer the same biological question as the algorithm is intended to do. This means that the same feature in the same image has to be measured by an independent and most possibly objective way. To explore this approach, we are performing a study (prepared for publication) which assesses the accuracy of two independent image analysis algorithms as compared to pathologist's measurement of the proportion of Ki-67-positive cells in breast cancer tissue that is immunohistochemically stained. The reference values were obtained by 3 observers independently on the same images using a stereological frame counting method to enumerate profiles of structures on 2D sections [14]. Initial findings reveal that the correlations between the two computer algorithms are very strong; they also correlate strongly with semiquantitative pathologist's evaluations. Meanwhile, the inter-observer variability in the stereologic counting is negligible. We found that the algorithms as well as pathologists may under- or overestimate numeric expressions of a biomarker when compared to the reference values obtained by the direct stereological estimation. Since the comparison of the crude results obtained can expose potential biases of both the digital image analysis tools and human visual perceptions, we believe this approach provides a sound methodology for evaluation, validation, and calibration of image analysis algorithms. A refined cell-to-cell control can then provide an estimation of the sensitivity and specificity of the digital analysis tools and a way to fine tune the algorithms. Therefore, stereology methods rather than pathologist's visual impressions should be used to obtain reference values. For use in daily practice as "quantitative tissue controls" reference images/slides will be needed for proper calibration and quality assurance of the image analysis tools.

In summary, digitization of pathology images brings a potential for the improvement of the quality of the information retrieved from the pathology samples. It enables the perspective of automation and precision of the tissue-based diagnosis and quantification. How- ever, digital image analysis can and should ensure accuracy and not just precision. To achieve the benefits of digitization, pathologists have to understand and assume new obligations in the quality assurance of the image analysis tools. Proper adoption of the technology will require a new quantitative mentality in pathology. In order to see a complete and sharp picture of a disease, pathologists will need to learn to use both their analogue and digital eyes.

\section{Acknowledgments}

This study has been partly supported by the COST Action IC0604, COST-STSM-ECOST-STSMIC0604-130211-005942 (Short-Term Scientific Mission, A. Laurinavicius), and the Research Council of Lithuania (A. Laurinaviciene).

\section{References}

[1] D. Soenksen, Digital pathology at the crossroads of major health care trends: Corporate innovation as an engine for change, Arch Pathol Lab Med 133 (2009), 555-559.

[2] J.O.R. Gu, Virtual microscopy and virtual slides in teaching, diagnosis and research in: Advances in Pathology, Microscopy \& Molecular Morphology, Series Editors J. Gu and Hacker GW Taylor \& Francis Group, Boca Raton, London, New York, Singapore (2005).

[3] L. Mulrane, E. Rexhepaj, S. Penney, J.J. Callanan and W.M. Gallagher, Automated image analysis in histopathology: A valuable tool in medical diagnostics, Expert Rev Mol Diagn 8 (2008), 707-725.

[4] A.M. Marchevsky, Image analysis, A primer for pathologists in: Raven Press, New York (1994).

[5] P.N. Furness, N. Taub, K.J.M. Assmann, G. Banfi, J.P. Cosyns, A.M. Dorman, C.M. Hill, S.K. Kapper, R. Waldherr, A. Laurinavicius, et al, International variation in histologic grading is large, and persistent feedback does not improve reproducibility, Am J Surg Path 27 (2003), 805-810.

[6] J.P.A. Baak, Manual of quantitative pathology in cancer diagnosis and prognosis in: Springer-Verlag, Berlin, Heidelberg, New York 1991.

[7] Joint Committee for Guides in Metrology (JCGM), International vocabulary of metrology: Basic and general concepts and associated terms (VIM), JCGM (2008), Available from: http://www.bipm.org/en/publications/guides/vim.html.

[8] P.J. Tadrous, On the concept of objectivity in digital image analysis in pathology, Pathology 42 (2010), 207-211.

[9] A. Nassar, C. Cohen, S.S. Agersborg, W. Zhou, K.A. Lynch, E.R. Heyman, A. Olson, H. Lange and M.T. Siddiqui, A new immunohistochemical ER/PR image analysis system: A multisite performance study, Appl Immunohistochem Mol Morphol 19 (2011), 195-202. 
[10] M.C. Lloyd, P. Allam-Nandyala, C.N. Purohit, N. Burke, D. Coppola and M.M. Bui, Using image analysis as a tool for assessment of prognostic and predictive biomarkers for breast cancer: How reliable is it? J Pathol Inform 1 (2010), 29.

[11] J. Slodkowska, V. Filas, E. Buszkiewicz, P. Trzeciak, M. Wojciechowski, R. Koktysz, W. Staniszewski, J. Breborowicz and M.G. Rojo, Study on breast carcinoma Her2/neu and hormonal receptors status assessed by automated images analysis systems: ACIS III (Dako) and scan scope (Aperio), Folia Histochem Cytobiol 48 (2010), 19-25.

[12] I. Skaland, I. Ovestad, E.A. Janssen, J. Klos, K.H. Kjellevold, T. Helliesen and J.P. Baak, Comparing subjective and digital image analysis HER2/neu expression scores with conventional and modified FISH scores in breast cancer, J Clin Pathol 61 (2008), 68-71.

[13] S. Vranic, B. Teruya, S. Repertinger, P. Ulmer, J. Hagenkord and Z. Gatalica, Assessment of HER2 gene status in breast carcinomas with polysomy of chromosome 17, Cancer 117 (2011), 48-53.

[14] T.M. Mayhew, The new stereological methods for interpreting functional morphology from slices of cells and organs, Exp Physiol 76 (1991), 639-665. 


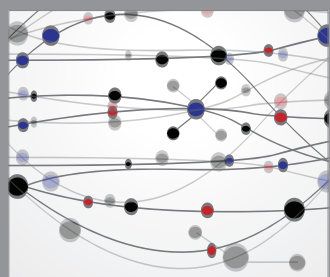

The Scientific World Journal
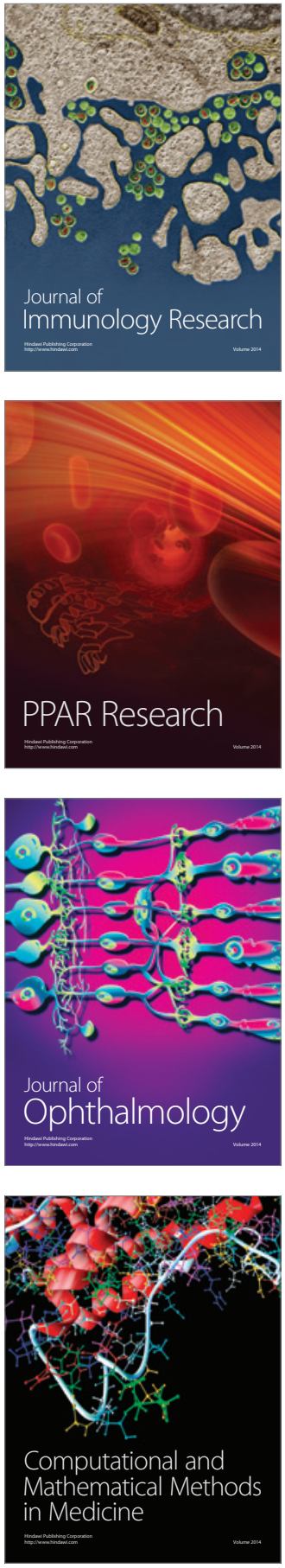

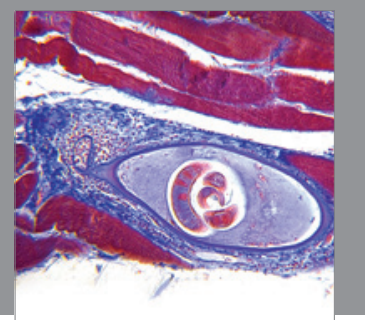

Gastroenterology

Research and Practice
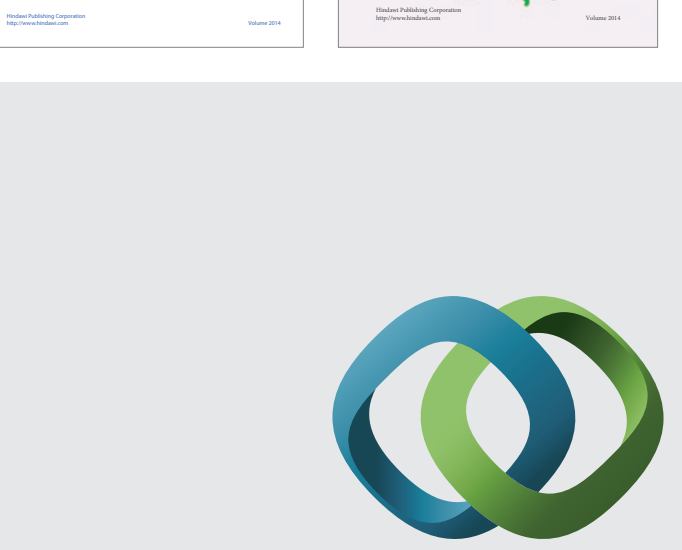

\section{Hindawi}

Submit your manuscripts at

http://www.hindawi.com
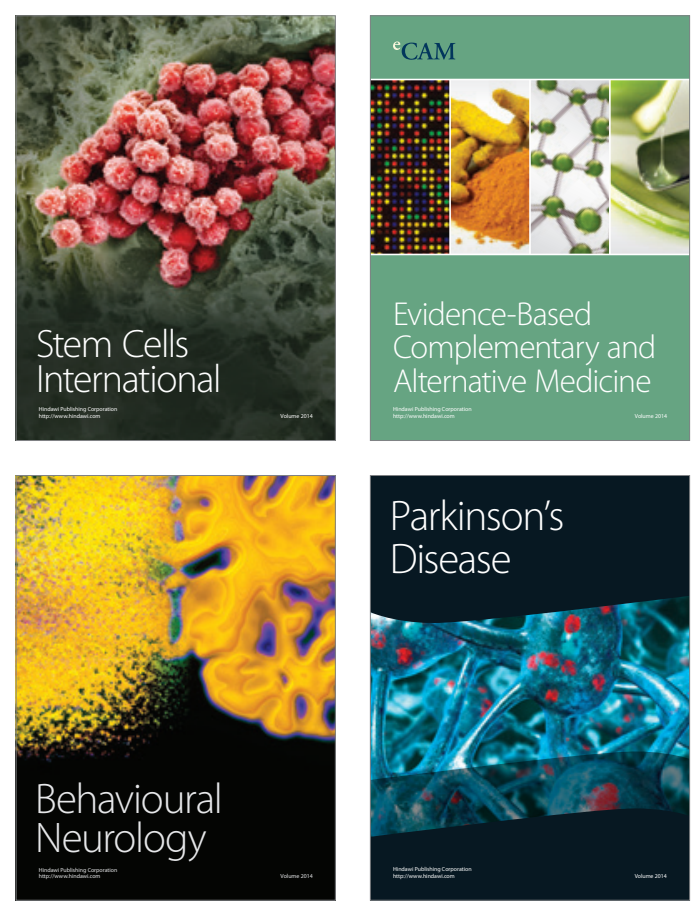

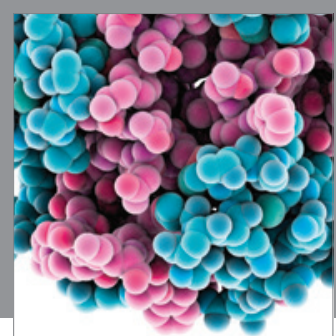

Journal of
Diabetes Research

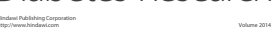

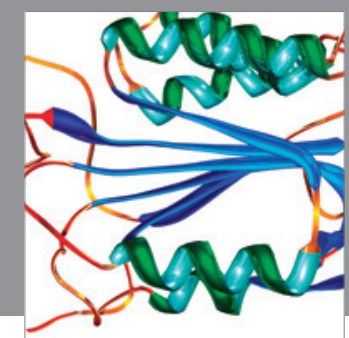

Disease Markers
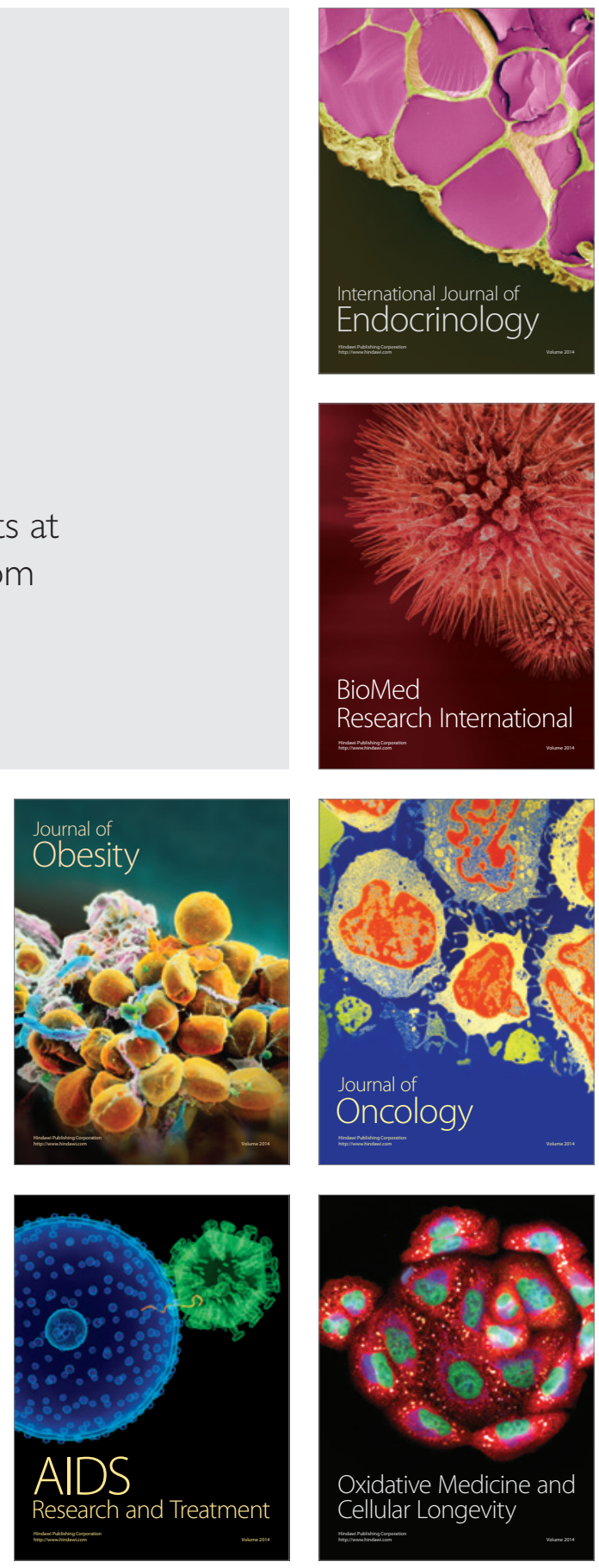